\title{
La universidad y la crisis ambiental: discursos y debates en el Perú
}

\author{
The university and the environmental crisis: \\ discourses and debates in Peru
}

Walter V. Castro Aponte ${ }^{1 *}$ Ricardo A. Yuli Posadas ${ }^{2}$

${ }^{1}$ Universidad Continental, Huancayo, ${ }^{2}$ Universidad Nacional Mayor de San Marcos, Lima

\section{RESUMEN}

El ensayo analiza los discursos y debates sobre la estructuración de la cuestión ambiental en la universidad peruana e identifica obstáculos y oportunidades para una reforma ambiental. La inclusión de la cuestión ambiental es superficial y las ciencias sociales están desvinculadas de las ingenierías al momento de abordar lo ambiental. La enseñanza transversal e interdisciplinar es compleja, básicamente por el perfil sociocultural de la sociedad peruana. Hay un cuestionamiento creciente de la legitimidad de la universidad para generar conocimiento, pero la apertura de la universidad hacia el conocimiento ancestral se constituye en una oportunidad de realizar una reforma ambiental efectiva. Un tema ausente en el debate de la universidad y la crisis ambiental es la apertura de la universidad hacia otros tipos de conocimiento. Un país como el Perú - por extensión los países andinos - está llena de entendimientos, cosmovisiones, perspectivas y conocimientos sobre la relación sociedad-naturaleza de por lo menos 5000 años de vigencia y que la universidad no entienda el conocimiento ancestral, menos busque puentes metodológicos transdisciplinares entre el conocimiento formal y este conocimiento ancestral es una verdadera lástima. Hay la necesidad de conocimiento transdisciplinar no solo para la solución de problemas puntuales como la erosión de los suelos, la conservación de la biodiversidad, calidad de aire y los residuos sólidos, sino para la planificación ambiental y el diseño de un modelo sostenible del país.

Palabras clave: Crisis ambiental, discursos, reforma ambiental.

\begin{abstract}
The essay analyzes the discourses and debates on the structuring of the environmental isssue in the peruvian university and identifies obstacles and opportunities for an environmental reform. The inclusión of the environmental issue is superficial and the social sciences are disconnected from engineering when addressing the environmental. Cross disciplinary and interdisciplinary teaching is complex, basically because of the socio cultural profile of Peruvian society. There is a growing questioning of the legitimacy of the university to generate knowledge, but the opening of the university towards ancestral knowledge constitutes an opportunity to carry out an effective environmental reform. A missing topic in the debate of the university and the environmental crisis is the opening of the university towards other types of knowledge. A country like Perú - by extensión the andean countries - is full of understandings, world views, perspectives and knowledge about the society - nature relationship of at least 5000 years of validity and that the university does not understand the ancestral knowledge, less seek methodological bridges transdisciplinary between formal knowledge and this ancestral knowledge and this ancestral knowledge is a real shame. There is a need for transdisciplinary knowledge not only for the solution of specific prblems such us soil erosion, biodiversity conservation, air quality and solid waste, but also for environmental planning and the design of a sustainable model of the country.
\end{abstract}

Keywords: Environmental crisis, speeches, environmental reform.

Historial del artículo:

Recibido, 04 de octubre de 2016; aceptado, 10 de junio de 2017; disponible en línea, 25 de junio de 2017

* Doctor en Política Ambiental por la Universidad de Wageningen, Holanda, Investigador de la Universidad Continental, Perú

Correo: kuntur.runa@gmail.com 


\section{INTRODUCCIÓN}

A inicios del siglo XXI nos encontramos en una situación donde la contaminación ambiental y la degradación de los ecosistemas son cada vez más intensos y a escala planetaria. Se ha establecido que la problemática ambiental no es un tema solamente ecológico sino multidimensional, con implicancias económicas, sociales, culturales, políticas, educativas e institucionales. En este sentido, cuestiona nuestros estilos de vida y nos invita a repensar nuestra valoración objetiva y subjetiva de la naturaleza. Esta situación de magnitud y escala sin precedentes que actualmente la sociedad humana experimenta ha sido referida por varios autores (Redclift, 2014; Sim, 2010; Beck, 2009; Oosthoek y Gills, 2005; Giddens, 2003) como crisis ambiental.

El Perú afronta problemas ambientales críticos de cambio de uso de suelos, deforestación, contaminación ambiental, y mala gestión de los residuos sólidos y vertidos líquidos (MINAM, 2012). Según The World Bank (2007), la contaminación ambiental y la vulnerabilidad a los desastres naturales tienen un costo económico del 3,9\% del producto bruto interno (PBI) del país. Asimismo, Lima ha sido calificado como la ciudad con el aire más contaminado de América Latina (EFE, 2014) y que a nivel nacional solo el $38 \%$ de los residuos sólidos son dispuestos en rellenos sanitarios (MINAM, 2014). Esta situación afecta a toda la población del país, indistintamente del poder adquisitivo y la ubicación geográfica. Los residuos de agroquímicos en los productos agrícolas, los compuestos orgánicos volátiles (COV) y compuestos orgánicos persistentes (COP) en el agua de consumo humano, las partículas en suspensión menores de 2.5 micras (PM 2.5) y 10 micras (PM 10) en el aire, los conflictos socio-ambientales, la inestabilidad política, entre otros, son situaciones íntimamente vinculados a la crisis ambiental del país. Si bien esta situación es crítica, no es un tema reciente. A lo largo de la historia colonial y republicana el auge y el colapso económico han estado directamente relacionados a la extracción de algún recurso natural, en algunos casos ocasionando la depredación del recurso como el caso de la anchoveta durante los 1960s y 1970s (World Bank, 2007).

A nivel de América Latina y El Caribe el panorama no es distinto. El Global Environmental Outlook (GEO) resalta el riesgo de los patrones de producción y consumo no solo para la conservación ambiental sino para el desarrollo de una economía sostenible. Esto está vinculado, como lo establece el GEO, a la creciente urbanización, la mayor proporción (79\%) de población urbana de las regiones en desarrollo del planeta, y a la escasa sensibilidad de la sociedad ante la crisis ambiental (PNUMA, 2010).
Ante esta situación apremiante la universidad no puede estar al margen, especialmente por su rol formativo y reflexivo en la sociedad. En efecto, la universidad es la institución que tiene por finalidad impartir educación superior, realizar investigación y proyección social y extensión universitaria, proporcionando recursos humanos calificados para la dinámica moderna. Según la nueva Ley Universitaria peruana, Ley № 30220, en su artículo 3 o establece: "La universidad es una comunidad académica orientada a la investigación y a la docencia, que brinda una formación humanista, científica y tecnológica con una clara conciencia de nuestro país como realidad multicultural. Adopta el concepto de educación como derecho fundamental y servicio público esencial. Está integrada por docentes, estudiantes y graduados. Participan en ella los representantes de los promotores, de acuerdo a ley". En la práctica, en el Perú universidad es un nombre genérico para una entidad altamente heterogénea, público o privada, de calidad educativa y capacidad académica diversa y que realiza una labor meramente instructiva, de formación profesional y/o de formación científica. De hecho, en el Perú la mayoría de las universidades están orientadas a la formación profesional y a la labor de consultaría, siendo la formación e investigación científica secundaria, marginal o nula. Esta situación se contrasta con el hecho que el Perú es el segundo país en Sudamérica con más universidades. Al 2013 existen en el país 140 universidades, 51 públicas y 89 privadas, lo cual muestra que en solo 13 años el número de universidades en el Perú prácticamente se ha duplicado (Macroconsult, 2014).

Desde la década de los 1990s se ha intensificado una serie de demandas a las universidades del país, tales como una mayor efectividad en su misión en la sociedad, respuestas oportunas a los grupos de interés y la generación de recursos económicos para financiar su funcionamiento. Demandas como la acreditación de la calidad es una cuestión de sobrevivencia para las universidades en un mercado no solo de mayor demanda de educación superior sino también de mayor oferta. Esto ha llevado a las universidades a un mayor uso de criterios de eficiencia y productividad empresarial para gestionar recursos propios $y$, a la vez, una orientación cortoplacista de satisfacer la demanda de profesionales en el mercado nacional. El acceso a la universidad se ha masificado en el país pero se evidencia dudas respecto a la calidad académica y educativa. Por otro lado se evidencia un contraste marcado de disponibilidad presupuestaria, infraestructura y calidad académico entre universidad privada y universidad pública. Vallaeys (2014) y otros autores han discutido las implicancias de estos cambios para la universidad, tales como la mercantilización de la educación superior y el cuestionamiento de su legitimidad.

Bajo este marco, tanto de crisis ambiental como de 
necesidad de cambio de la universidad, el ensayo aborda las siguientes interrogantes: ¿̇Cómo la universidad responde a la crisis ambiental y la necesidad de sostenibilidad? ¿Cuáles son los discursos y debates al respecto? ¿Qué contribuciones podemos brindar al debate ambiental a nivel internacional desde la experiencia peruana? En este sentido, el ensayo brinda luces sobre la institucionalización de la cuestión ambiental en la universidad y la necesidad de reforma ambiental, tomando como referencia la universidad peruana.

\section{Más de un discurso sobre cómo integrar la cuestión ambiental en la universidad}

La universidad no es un espacio social uniforme ni libre de discrepancias en el abordaje de la cuestión ambiental, sino un espacio de disputa y tensiones de posturas. Estas discrepancias en posturas sobre la relación sociedad-naturaleza son codificadas en discursos. Los discursos ambientales son entendimientos y visiones sobre la relación sociedadnaturaleza basadas en supuestos, juzgamientos y contradicciones que permiten el análisis, el debate y la identificación de coincidencias y discrepancias (Dryzek, 2005; Castro, 2013). Los discursos ambientales de la universidad se estructuran en base a los puntos de vista de estudiantes, docentes y autoridades sobre la problemática ambiental y las estrategias de abordaje tanto en los estamentos de gestión institucional, docencia e investigación, y proyección social y extensión universitaria. En este sentido, los discursos ambientales de la universidad establecen la posición institucional de la comunidad universitaria no solo objetiva sino subjetiva, no solo formal sino no formal, no solo explicita sino implícita frente a la problemática ambiental.

Hay más de un discurso sobre cómo la cuestión ambiental se estructura en la universidad y cuál estrategia es la más eficaz para responder a la crisis ambiental. Mientras que algunos sostienen que el reconocimiento de la crisis ambiental actual y el cambio de la universidad hacia la sostenibilidad parte desde un sentido del "deber" institucional, otros sostienen que es el resultado natural de la "conciencia" colectiva universitaria. En cuanto al abordaje de lo ambiental en la universidad, algunos priorizan el enfoque académico monodisciplinar y de especialización mientras que otros priorizan el enfoque interdisciplinar y transversal. Algunos le dan mayor atención a la formación profesional, mientras que otros enfatizan la acción social. Mientras que algunos resaltan la implementación de proyectos ambientales a pequeña escala, otros prefieren a gran escala. Algunos enfatizan el conocimiento tecnológico en el abordaje de la problemática ambiental, otros el conocimiento social, y finalmente, mientras que algunos sostienen que la promoción del empresarialismo es la manera más efectiva de responder a la crisis ambiental, para otros es la promoción del voluntariado.

A continuación analizamos estos discursos a más a detalle.

\section{La protección ambiental: ¿̇Deber o conciencia?}

El discurso del "deber" ambiental y la "conciencia" ambiental son dos miradas distintas de la universidad a la crisis ambiental. "Deber" es entendido como "compromiso", "obligación" en beneficio de la sociedad. Los proponentes de este discurso utilizan comúnmente la expresión "tengo que." "tenemos que." para mostrar su adhesión. En este discurso el estamento de gestión institucional tiene rol prominente al establecer las directivas de incorporación de la preocupación ambiental en la universidad. La incorporación se da a nivel de la formación profesional, la proyección social y la investigación. Por otro lado, el discurso de la conciencia ambiental resalta la convicción y la iniciativa individual. Según los proponentes de este discurso la mayor preocupación ambiental de la universidad no es el resultado de reglas y normas sino del compromiso y la actitud proactiva de los miembros. Los proponentes se inclinan por actividades de activismo ambiental y campañas de educación ambiental.

Principal responsabilidad de la universidad: ¿̇Formación profesional o acción social?

El discurso de la formación profesional prioriza la enseñanza e instrucción estandarizada del estudiante basado en estructuras pedagógicas rígidas y el establecimiento de competencias previamente establecidas por las autoridades de la universidad. Por otro lado, el discurso de la acción social enfatiza la capacidad de influencia de la universidad en la sociedad, más que en labores netamente instructivas. La acción social puede ser variada. En algunos casos es mediante la investigación aplicada de un problema ambiental determinado, en otros casos es mediante la participación de los miembros de la comunidad universitaria en actividades de extensión y proyección social de la propia universidad.

El énfasis en la acción social se enmarca como parte del movimiento de la responsabilidad social de la universidad. La responsabilidad social es un movimiento social de origen filantrópico y empresarial que en los últimos años se ha extendido a la universidad canalizando los esfuerzos de activismo ambiental de los miembros de la comunidad universitaria. Al respecto en los últimos años la responsabilidad social y la iniciativa empresarial han sido promovidas activamente por las universidades peruanas en términos de competencias. La Universidad del Pacifico, la Pontificia Universidad Católica del Perú, la Universidad Peruana Cayetano 
Heredia y la Universidad Continental son las que han mostrado mayor dinamismo. Sin embargo, la acción social también ha tomado formas más "sagaces" de proyección social en el Perú mediante becas y campañas sociales gratuitas con fines distintos a lo estrictamente educativo. Este es el caso del consorcio de universidades privadas (Universidad César Vallejo, Universidad Señor de Sipán y Universidad Autónoma del Perú) que se ha constituido en una plataforma electoral para el dueño del consorcio César Acuña para las elecciones a la presidencia del Perú.

¿Cuál conocimiento es determinante para la sostenibilidad, lo tecnológico o lo social?

El conocimiento tecnológico enfatiza la capacidad de cálculo, medición y operación, el aprendizaje práctico y el abordaje de problemas concretos. Es decir, enfatiza las competencias "duras" que permiten diseñar, hacer, crear y fabricar, incluyendo el proceso, los procedimientos y el control de los mismos con el fin de satisfacer necesidades e intereses. La tecnología se constituye en recurso indispensable para dar valor al recurso natural que permita generar una dinámica económica-productiva eficiente. Este primer discurso resalta la importancia del diseño, los diagnósticos, el análisis de procesos, la valoración cuantitativa y la medición de la calidad. Por lo que la lectura de la problemática ambiental es mecanicista, por lo usual en términos de causa - efecto. Por esta razón, los proponentes resaltan la importancia de las ingenierías, la administración de empresas, y en parte, de las ciencias naturales y médicas, pero infravalora las ciencias sociales, en especial la sociología y la antropología los cuales son vistas como innecesarias. En este discurso las humanidades y las artes son vistas como inútiles.

El discurso tecnológico comprende dos variantes. Una vinculada con el conocimiento de las ciencias naturales, la agropecuaria y los negocios, y otra vinculada con el conocimiento estructural, la infraestructura y el diseño. El discurso tecnológico separa lo ambiental en ingeniería ambiental y ecologismo. El ecologismo es visto como activismo político que promueve la conservación de la naturaleza desde una posición radical. En cambio, la ingeniería ambiental busca el desarrollo, uso y aprovechamiento sostenible de los recursos naturales. En este sentido, si bien identifica la complementariedad entre la protección ambiental y la eficiencia productiva, por ejemplos en términos de negocios limpios y responsables, este discurso no visualiza las tensiones, y conflictos socioculturales como resultado de la intervención tecnológica. Bajo esta lente las tensiones son solo el resultado de la aplicación incorrecta de algún método o instrumento técnico. Este discurso ignora las relaciones de poder, los intereses, las cuestiones culturales e interculturales, la participación social, entre otros aspectos cualitativos.
En este sentido la gestión ambiental es entendida como una "caja de herramientas" y la cuestión social es reducida a la implementación de reuniones, talleres y jornadas con los beneficiarios o clientes con fines informativos.

Por otro lado, el discurso del conocimiento social pone en primer plano el conocimiento de las ciencias sociales y el análisis de la realidad social humana. En este discurso son visibles los marcos conceptuales, ideológicos, históricos, valorativos, relaciones de poder, imaginarios sociales y otros aspectos objetivos y subjetivos de las relaciones humanas. Es usual el análisis de estructura social y la capacidad de agencia. Esta perspectiva enfatiza las competencias "blandas" y habilidades sociales, permitiendo desarrollar el espíritu crítico del estudiante y su posicionamiento. Bajo este discurso el conocimiento tecnológico es visto mecánico y repetitivo para el estudiante. Con la emergencia de los conflictos ambientales y la falta de entendimiento intercultural se ha visto con más urgencia la necesidad del conocimiento social para entender la problemática ambiental del país.

¿Abordar la cuestión ambiental desde la transversalización o la especialización?

Transversalidad y especialización son dos discursos contrapuestos en el abordaje de la cuestión ambiental. El enfoque transversal resalta el carácter complejo de la problemática ambiental y la necesidad de abordarlo integralmente desde diferentes aristas y miradas. Se asume que el abordaje distributivo de las competencias ambientales en diferentes cursos y actividades curriculares y extracurriculares permite un abordaje más efectivo de la problemática ambiental. El enfoque transversal permite ampliar el espectro de posibilidades de análisis de la problemática ambiental creando puentes y dialogo entre distintas áreas del conocimiento, distintas carreras y distintas facultades de la universidad. La transversalidad permite que lo ambiental se difunda a todas las áreas del conocimiento generando retroalimentación y fertilización cruzada.

Los proponentes de la transversalidad aducen que este enfoque permite mayor flexibilidad en la formación ambiental del estudiante, indistintamente de la carrera que estudia, permitiéndole una mayor capacidad de adaptación a la incertidumbre y los escenarios cambiantes. En este discurso, la especialización es visto como conocimiento fragmentado, con paradigmas y enfoques distintos, disimiles y en ocasiones contradictorias. Por lo tanto, la transversalidad enarbola lo interdisciplinar.

Por otro lado, la especialización enfatiza la división del conocimiento en áreas o disciplinas académicas, tales como ecología, economía, sociología, derecho, medicina, educación, administración. En el Perú se 
suelen llamar carreras profesionales. La especialización está delimitada por paradigmas, enfoques y campos de acción definidos. En este discurso, lo ambiental es visto emparentado solo con lo ecológico y separado de lo social, económico y educativo. Lo ambiental es un ángulo más de análisis de la realidad que compite e inclusive contradice otras áreas del conocimiento. En este sentido, el desencuentro entre ecología y economía es usual. Ahora, si se da un abordaje ambiental esta se restringe al marco conceptual de la disciplina específica, pero no se da un análisis interdisciplinar. Este es el caso del derecho ambiental que discute la cuestión ambiental desde la óptica del derecho y la ingeniería ambiental que aborda la cuestión ambiental desde la óptica de los procesos y las tecnologías. En este discurso, el abordaje integral de la cuestión ambiental no es prioritario, sino el estudio puntual.

Por lo tanto, el discurso de la especialización enfatiza lo monodisciplinar y las competencias a lograr por los estudiantes están restringidas a un área del conocimiento específico. Los proponentes aducen que la división del conocimiento en disciplinas permite ganar mayor profundidad en el análisis y ser más efectivo en la solución de un problema específico y el abordaje de la realidad, y no menos importante, tener mayores posibilidades de acceder a una plaza laboral.

\section{¿̇"Lo pequeño es hermoso" o "lo grande es mejor"?}

La priorización de proyectos ambientales a pequeña escala es usualmente parte de la labor filantrópica de la universidad o es un complemento de los cursos académicos con el fin de familiarizar al estudiante con contextos socioculturales que no le es propio. Los proyectos a pequeña escala suelen ser jornadas, talleres, marchas, campañas, plantones, entre otros. Los proponentes de este discurso suscriben la frase "lo pequeño es hermoso" (proviene de la frase en inglés "small is beautiful") para resaltar que una acción pequeña tiene mayor significancia en la formación profesional del estudiante universitario.

En este sentido, los proponentes de acciones ambientales a pequeña escala consideran que la experiencia vivencial y el contacto humano son centrales para superar la actual crisis ambiental en el país. En cambio, la priorización de proyectos ambientales de gran envergadura está orientado a abrir nuevos frentes de "negocio" de la universidad y una mayor relación con los grupos de interés. En estas iniciativas no solo participan estudiantes, egresados y docentes sino expertos afiliados a la universidad. Los proponentes de este discurso suscriben la frase "lo grande es mejor" (proviene de la frase en inglés "big is better") para resaltar que lo acción a gran escala tiene mayor impacto en la sociedad y la intervención a pequeña escala es marginal.
¿Cuál es la estrategia más efectiva para la sostenibilidad, empresarialismo o voluntariado?

El empresarialismo es un discurso que promueve la formación de profesionales competitivos y emprendedores, con iniciativa empresarial. En este sentido bajo este discurso, las necesidades del mercado y del emprendedor empresario tienen una posición gravitante. La perspectiva del empresarialismo tiene dos vertientes: un discurso centrado en la competitividad económica, la rentabilidad y la creación de valor económico y el otro más holístico, incluyendo lo social y ambiental, la investigación científica y la generación de valor social. En el primer caso, se da atención prioritaria a las unidades de negocio, que generan ingresos económicos y la sostenibilidad no es tomada en cuenta. En el segundo caso, se valora las consideraciones sociales y ambientales en los negocios; tales como los negocios "inclusivos", negocios "sostenibles", "Fair Trade". En este último caso, la competitividad considera las normas laborales, ambientales y de salud pública, la dinamización de la economía local y la responsabilidad social. Los proponentes del empresarialismo promueven la participación e integración a los procesos globales, ser parte de sus dinámicas y gozar de sus beneficios.

Por otro lado el voluntariado es entendido como la acción ambiental de los estudiantes universitarios u otros miembros de la universidad dentro y fuera del espacio físico universitario. Es decir, el voluntariado no se restringe a la universidad ni a la participación en asuntos institucionales de extensión universitaria y de proyección social de la universidad, sino se extiende a las Organizaciones No Gubernamentales (ONG), asociaciones civiles, clubes juveniles, asociaciones profesionales, organización de base, entre otros. El discurso del voluntariado promueve la promoción de la educación ambiental, y defensa del patrimonio cultural y ambiental. A diferencia de la acción social que es planificada y sistemática, el voluntariado enfatiza lo vivencial, lo espontaneo y la creación de "valor social". Los proponentes del discurso del voluntariado se orientan a la transformación social y hacen énfasis en la generación de valor más que el lucro. Asimismo, no son muy asiduos a enfatizar el emprendedurismo sino la acción voluntaria.

\section{Avances y retos en la integración de la cuestión ambiental en la universidad peruana}

Un número creciente de universidades están incluyendo la cuestión ambiental en la gestión, formación, investigación, extensión universitaria y proyección social de la universidad. La inclusión se evidencia por la referencia a una serie de términos en el plan estratégico institucional que denotan la preocupación 
ambiental; tales como "medio ambiente", "ecológico" "ambiental", "desarrollo sostenible", "sostenibilidad", "desarrollo ambiental" "responsabilidad social", entre otros. Otra evidencia es la incorporación de cursos de medioambiente, ecología o equivalentes, ya sea como cursos generales para todos los estudiantes - como cursos especializados, y el incremento significativo de los proyectos de investigación científica con enfoque ambiental. Asimismo, en los últimos años la universidad peruana a mostrado una mayor preocupación social con iniciativas de corte ambiental a través de la extensión universitaria y la proyección social. Estos avances se han dado mayormente en las universidades privadas, tales como la Universidad del Pacifico, la Pontificia Universidad Católica del Perú, la Universidad Peruana Cayetano Heredia y la Universidad Continental. Un avance significativo en la institucionalización de la cuestión ambiental en la universidad peruana es la formación de la Red Ambiental Interuniversitaria - Interuniversia Perú (RAI). Con una trayectoria de más de quince años, RAl agrupa a 71 universidades del país que aspiran a ser ejemplo de responsabilidad ambiental.

Sin desmerecer estos avances es preciso destacar que la inclusión de la cuestión ambiental en la universidad peruana es escasa, superficial, parcial y segmentada. Los cursos implementados son meramente instructivos, narrativos y no cuestionan el estilo de vida de los grupos de interés de la universidad, menos generan debate ni ejercen una influencia en la sociedad pervana. Es más, la inclusión de cursos de corte ambiental no necesariamente ha significado la integración del concepto a nivel institucional, menos aun de un cambio institucional sustancial de la universidad.

La universidad presenta debilidades en la enseñanza transversal de las ciencias sociales y las ciencias ambientales, ambos pilares para el entendimiento de la problemática ambiental. Las ciencias sociales se enseñan de manera tradicional, segmentada, escasamente aplicada y la investigación científica a nivel de análisis teórico social de la cuestión ambiental es escasa. Si bien se han incluido un curso de ecología o afín en el plan de estudios, la cuestión ambiental no es gravitante en la agenda institucional y la formación universitaria. $Y$ si es gravitante, como en el caso de la carrera de ingeniería ambiental, la cuestión ambiental se limita a lo tecnocrático y divorciado de sus componentes sociales y culturales. Por lo usual lo social y lo ecológica son abordados de manera separada tanto en el plan de estudios como en la gestión institucional. No hay cursos específicos en el plan de estudios o espacios académicos que permitan discutir sistemáticamente la relación entre lo social, lo ecológico, lo económico y otros ángulos de análisis. Menos aún, que se genere conocimientos en base a estas discusiones. La enseñanza interdisciplinar es mal entendida. Se asumen que poner estudiantes de distintas disciplinas en un salón de clases o que realicen labores de aprendizaje conjuntas es interdisciplinar. Esto es multidisciplinariedad, pero no interdisciplinariedad. No se desarrolla las capacidades del estudiante para abordar la cuestión ambiental y de la sostenibilidad con integridad. En este sentido se evidencia, por un lado, la necesidad de rediseñar la enseñanza de las ciencias sociales, y por el otro, la necesidad de una apertura de las ingenierías y los estudios empresariales a las ciencias sociales con el fin de lograr un abordaje interdisciplinar de la relación sociedad-naturaleza.

La operacionalización de la cuestión ambiental a nivel de la toma de decisiones y su implementación interdisciplinar a nivel del plan de estudios es un tema pendiente en el país. Las razones son varias. Por un lado, no existe aún un consenso sobre en qué consiste realmente una universidad "sostenible", una universidad "ecológica" ○ una universidad "socialmente responsable" y sobre cuál es la estrategia de implementación y gestión más acertada para que la universidad experimente cambios significativos hacia la sostenibilidad.

Es preciso mencionar que algunas universidades están haciendo un avance en la enseñanza transversal del conocimiento ambiental, como es el caso de la Universidad Continental, sin embargo, se ha observado la resistencia a nivel de decanos y docentes de las escuelas académicas de ingeniería y empresas para incluir cursos de sociología y ecología en sus programas académicos. Sumado a esto, es preciso considerar la presión actual del mercado laboral peruano por profesionales con experticia tecnológica monodisciplinar.

Otro reto que está en el centro del debate del diseño de las políticas de educación superior es la escasa investigación científica. La mayoría de los docentes no tienen el perfil ni la motivación para ello. La escasa investigación que se realiza es monodisciplinar, individual y aislada. No es usual la investigación interdisciplinar, menos transdisciplinar, y la aplicación de enfoques y metodologías actuales en el análisis de la problemática ambiental. Menos aún se estimula la revisión y síntesis de lo investigado. Por otro lado, la formación del estudiante en investigación es deficiente. Sin embargo, no se trata solamente de incrementar el número de tesis universitarias sino orientarlo hacia el abordaje interdisciplinar y transdisciplinar. Las causas de esta deficiencia son múltiples; entre otros la falta de interés de los estudiantes, la falta de una agenda de investigación ambiental, la falta de una estructura de suporte académico y económico, y no menos importante, la demanda de un mercado laboral por profesionales "operativos" y no necesariamente científicos. Hay preguntas pendientes por absolver: ¿Qué estrategias utilizamos para revertir esta situación? ¿QQué incentivos? ¿Cómo generamos 
espacios de reflexión académica? ¿Cómo generamos equipos interdisciplinares y transdisciplinares? ¿̇Cómo hacer investigación con grupos de estudiantes tesistas organizados en "clusters" de investigación? La investigación científica es fundamental si la universidad desea no solo posicionarse en el mercado de la educación superior sino cumplir con su razón de ser.

Un tema usualmente no discutido es el hecho que las prioridades de investigación no están determinadas necesariamente por su relevancia sino por la disponibilidad de financiamiento, que usualmente proviene de contrapartes y donantes internacionales. Es más, las prioridades establecidas por los organismos internacionales y el gobierno nacional no necesariamente son las prioridades de la población, especialmente a nivel local. $\dot{2}$ En base a que evidencias científicas podemos afirmar que determinado tema es prioritario sobre otro a nivel local? Por ejemplo, ¿Es prioridad el cambio climático o la gestión sostenible de los residuos sólidos? ¿̇Es prioridad la contaminación atmosférica urbana o la conservación de la biodiversidad? ¿̇Es prioridad la erosión de suelos o el modelamiento climático? ¿̇Es prioridad desarrollar nuevas tecnologías de producción sostenible o poner en valor los conocimientos de la agricultura ancestral? ¿Quién establece estas prioridades? ¿̇cómo se integran estas prioridades a nivel local?

Un reto no menos importante de la universidad es el cuestionamiento de su legitimidad como generador $y$ diseminador del conocimiento para la sostenibilidad, es decir el cuestionamiento de las bases cognitivas y epistemológico que sostienen la educación superior que proporciona la universidad. ¿Cuál es el impacto cognitivo y epistemológico del accionar académico de las universidades en la sociedad? ¿Cuál es la contribución de los miles de profesionales que egresan de las universidades a la resolución de la crisis ambiental? ¿́La universidad forma a sus estudiantes, indistintamente del programa académico, para solucionar la crisis ambiental? ¿Para administrar la crisis ambiental? o ¿Para actuar con indiferencia ante la crisis ambiental? El abordaje de estas cuestiones centrales pasa por el avance de lo monodisciplinar hacia lo interdisciplinar, una re-definición del rol social de la investigación científica que este a la altura de la crisis ambiental que enfrentamos y una apertura de la universidad hacia el conocimiento no formal de la sociedad incluyendo sus diversidad en formas de entender y abordar la problemática ambiental.

En esta apertura es imperativo la creación de puentes de diálogo metodológico entre el conocimiento formal y el conocimiento ancestral. Un buen punto de partida es la exploración de la racionalidad ambiental desde la perspectiva de las sociedades ancestrales quienes han logrado avances destacados en el entendimiento de las subjetividades. Este diálogo metodológico tiene que ser en esencia intercultural y distar, por un lado, del discurso de "inclusión" que promueve una asimilación cultural de las sociedades ancestrales, y por el otro lado, de la "folclorización" del conocimiento ancestral. Ciertamente a la luz de la crisis ambiental actual no es viable esperar respuestas contundentes del modelo actual de universidad para responder con eficacia a la actual crisis ambiental; para este cometido la universidad tiene que re-inventarse.

\section{Hacia la reforma ambiental de la universidad}

Diversas investigaciones (Buttel, 2003; Mol, 2008; Mol, 2010, Castro; 2013) han argumentado que la crisis ambiental pone a prueba la capacidad de cambio de las instituciones de la modernidad. Reforma ambiental implica que las instituciones de la modernidad tienen la capacidad de resolver la crisis ambiental mediante el desarrollo tecnológico, el cambio de los procesos en la organización y de las prácticas de gestión ambiental estratégica. Si bien se ha analizado y discutido en qué medida el gobierno, el aparato productivo y las ONG están reformando sus estructuras bajo criterios ambientales, no se ha explorado que tanto las universidades, en su condición de institución pilar de la modernidad está reformándose ambientalmente.

La reforma ambiental de la universidad implica el desarrollo de tecnologías educativas, tecnología de la información y las comunicaciones (TICs) y modelos pedagógicos de integración disciplinar, interdisciplinar y transdisciplinar donde la sostenibilidad es el eje integrador. Asimismo, implica cambios en la estructura organizacional, plan de estudios y la implementación a nivel institucional de enfoques de vanguardia tales como análisis del ciclo de vida, ecoeficiencia, carbono neutral, ISO 26000, entre otros. Así como la universidad comparte con otros actores sociales de la modernidad aspectos comunes en la reforma ambiental, también presenta particularidades. La universidad es distinta en su naturaleza y fines al gobierno y a la empresa, siendo su principal misión la generación y transmisión de conocimiento. En este sentido, la reforma ambiental de la universidad peruana incluye cambios no solo en la manera y el propósito de la enseñanza universitaria sino en la apertura a la diversidad de conocimientos y perspectivas de la realidad.

En un intento por estar a la altura de la crisis ambiental, las universidades en el Perú han empezado a adoptar la cuestión ambiental a nivel de la gestión institucional, la educación que imparten, la investigación que realizan y la labor social que emprenden. En este sentido, las universidades están adoptando diversos conceptos que refieren a una re-definición de la relación de la sociedad con la naturaleza tales como "desarrollo sostenible", "sostenibilidad" y "responsabilidad social". Lo que es relevante es que la adopción de estos conceptos que 
expresan la vinculación directa de la universidad con la cuestión ambiental pone en la mesa la necesidad de cambios en la estructura institucional, el modelo educativo y las prioridades en la investigación. Es más, la inclusión de la cuestión ambiental ha puesto en debate los fundamentos mismos de lo que es una universidad, su rol en la sociedad y lo que definimos como conocimiento.

La promulgación de la nueva Ley Universitaria el año 2014 se visualiza como una oportunidad para enrumbar la universidad peruana hacia una reforma ambiental. Tanto en los artículos 6, 124 y 125 dicha ley hace referencia explícita a la cuestión ambiental al mencionar el "desarrollo sostenible" y la "responsabilidad social". Sin embargo, no establece con claridad el rol central de la universidad en la resolución de la crisis ambiental del país y la orientación de la educación superior hacia la sostenibilidad. Para que la universidad peruana experimente cambios significativos hacia la sostenibilidad, es imperativo abordar la débil institucionalidad, el escaso liderazgo ambiental y la limitada capacidad de influencia en la sociedad. En este sentido, el rol de la universidad es central no solo a nivel del replanteamiento de la educación sino también a nivel de la re-estructuración de otros pilares de la modernidad tales como el gobierno y el aparato productivo. La universidad tiene una posición privilegiada al ser la institución que replica la modernidad y con el potencial de enrumbarlo hacia la sostenibilidad.

Los esfuerzos de desarrollar el marco normativo, la estructura organizacional, los códigos éticos y la política ambiental institucional son solo el primer paso en la reforma ambiental de la universidad. No menos importante es el re-diseño orientado a la sostenibilidad del "software" de la universidad que viene a ser, en primer lugar, el equipo humano que lidera la institución, en segundo lugar, el plan de estudios, en tercer lugar, la agenda de investigación y por último, el perfil sociocultural del estudiante peruano. Especialmente el primer y el último factor constituyen un factor decisivo para cualquier cambio sustancial de la universidad. La sociedad pervana es ambientalmente insensible, prima los intereses de grupo en periuicio del bien común y las relaciones sociales están determinadas por valoraciones económicas y prejuicios étnico-raciales. Un reflejo emblemático de este perfil sociocultural es el "muro de la vergüenza" ubicado en la ciudad de Lima, capital del país. Mientras que para algunos es discriminación, para otros es solo una respuesta a la inseguridad (Pighi, 2015). Lo evidente es que el "muro de la vergüenza" es una muralla física que refleja las profundas contradicciones no resueltas del país.

Así como se presentan obstáculos también se visualizan oportunidades para la reforma ambiental de la universidad. El surgimiento de nuevos actores en la educación superior y la investigación científica tales como las "global universities", "open universities", "online universities" "universidades indígenas" y nuevas formas de educación tales como los "Massive Open Online Courses (MOOCs)", "e-learning", "field schools", están cambiando completamente el panorama de la educación superior en el país y la región de América Latina. Sin embargo, aún es muy pronto para dilucidar en que medida estos cambios tecnológicos y de procesos educativos de carácter global y regional pueden condicionar la reforma ambiental de la universidad peruana.

\section{CONCLUSIONES}

Identificar los discursos sobre cómo se estructura la cuestión ambiental, así como los avances y retos al respecto permite clarificar el panorama del debate ambiental en la educación superior. Queda establecido que no hay un discurso único sino un abanico de discursos con perspectivas particulares sobre la cuestión ambiental y su estructuración en la universidad. Los discursos analizados no necesariamente nos ponen un panorama de antagonismos sino de coincidencias en los puntos de vista, de complementariedad de perspectivas y de una construcción progresiva del discurso ambiental. Sin embargo, la indiferencia y la invisibilizarían de la crisis ambiental es también muestra de una posición en el debate ambiental. Es decir, no solo las posturas discrepantes son parte del debate ambiental, sino también la ausencia de posturas. Por lo tanto, la universidad no es una entidad imparcial sino encarna las tensiones y antagonismos de índole ideológico, político, social, económico, de cosmovisión y de poder de la sociedad peruana.

Los cambios que la universidad peruana está experimentando en términos de reforma ambiental son lentos, en cierta medida aleatoria, con escasas propuestas y sin dirección clara, mientras que la crisis ambiental es contundente y verificable. Si bien se ha referido repetidas veces a la necesidad de la interdisciplinaridad y transdisciplinariedad y la necesidad de afianzar la investigación científica para la sostenibilidad en la universidad, no se han tomado medidas contundentes al respecto ni desde la universidad misma ni desde el gobierno. La interdisciplinaridad y transdisciplinariedad en la universidad no es un asunto sencillo en el Perú, tampoco en América Latina, debido a que hay vacíos metodológicos y obstáculos contextuales. Se sigue pensando, por ejemplo, que la práctica del arte no tiene relación con el estudio de las ciencias, y que la filosofía y la ética son irrelevantes para la formación en ingenierías y gestión empresarial. El enfoque monodisciplinar esta tan arraigado no solo en las universidades sino en la sociedad peruana que los esfuerzos tienen que ir más allá del establecimiento del marco normativo de la Ley Universitaria. Cambiar de una formación monodisciplinar a una interdisciplinar 
orientada hacia la sostenibilidad, pasa por cambiar el "mindset" de la universidad y repensar la visión de país.

Finalmente, un tema ausente en el debate de la universidad y la crisis ambiental es la apertura de la universidad hacia otros tipos de conocimiento. Un país como el Perú - por extensión los países andinos - está llena de entendimientos, cosmovisiones, perspectivas y conocimientos sobre la relación sociedad-naturaleza de por lo menos 5000 años de vigencia y que la universidad no entienda el conocimiento ancestral, menos busque puentes metodológicostransdisciplinares entre el conocimiento formal y este conocimiento ancestral es una verdadera lástima. Hay la necesidad de conocimiento transdisciplinar no solo para la solución de problemas puntuales como la erosión de los suelos, la conservación de la biodiversidad, calidad de aire y los residuos sólidos, sino para la planificación ambiental y el diseño de un modelo sostenible del país. A la luz de la crisis ambiental que enfrentamos el aletargamiento de la universidad no solo es tema de debilidad institucional sino de limitación del mismo conocimiento tecnológico-científico formal. Queda por dilucidar si la aparición de nuevos actores en la educación superior y la visibilización de otras formas de conocimiento distinto al conocimiento formal coadyuvaran al colapso de la universidad o a su reforma. En este sentido, si avizoramos la vigencia de la universidad y su legitimidad en el siglo XXI es imperativo tomar en serio la reforma ambiental, que definitivamente pasa por la re-definición de la cuestión cognitiva - epistemológica y la creación intercultural del conocimiento para la sostenibilidad.

\section{REFERENCIAS BIBLIOGRÁFICAS}

Beck, U. (2009). World at Risk. Cambridge, UK:Polity Press.

Buttel , F. H. (2003). Environmental Sociology and the Explanation of Environmental Reform. Organization \& Environment.

Castro, W. (2013). Non-governmental organizations in promoting sustainability of small and mediumsized enterprises in Peru: An analysis of networks and discourses. Tesis para obtener el grado de Doctor en Política Ambiental, Wageningen University, Wageningen, The Netherlands. Obtenido de http://edepot.wur.nl/258818

Congreso de la Republica del Perú. (09 de julio de 2014). Ley $N^{\circ} 30220$ Ley Universitaria. Diario Oficial El Peruano. Obtenido de http:// diariooficial.elperuano.pe/Normas
Dryzek, J. S. (2005). The politics of the earth: environmental discourses. 2nd. New York: Oxford University Press.

EFE. (07 de mayo de 2014). Lima es la ciudad más contaminada de Latinoamérica. El Comercio. Obtenido de http://elcomercio.pe/ ciencias/planeta/lima-tiene-peores-nivelescontaminacion-latinoamerica-noticia- 1727740

Giddens, A. (2003). Runaway World: How Globalization is Reshaping Our Lives. New York, USA: Routledge.

Macroconsult. (05 de julio de 2014). El número de universidades en el Perú se duplicó en solo 13 años. Gestion. Obtenido de http://gestion. pe/economia/numero-universidades-peru-seduplico-solo-13-anos-2102202

Mol Arthur, P. J. (2010). Social Theories of Environmental Reform: Towards a Third Generation. En M. Gross y H. Heinrichs (Eds.), Environmental Sociology: European Perspectives 19 and Interdisciplinary Challenges. 19-38. USA: Springer.

Mol Arthur, P. J. (2008). Environmental reform in the information age: the contours of informational governance. New York, USA: Cambridge University Press.

Oosthoek , J., \& Gills, B. K. (2005). Humanity at the crossroads: The globalization of environmental crisis. 3(2), 283-291.

Perú, Ministerio del Ambiente del Perú. (2014). Agenda ambiental 2015-2016. Agenda nacional de acción ambiental. Obtenido de http://www.minam.gob. pe/wp-content/uploads/2014/12/ANEXO-I.pdf

Pighi, P. (22 de octubre de 2015). El polémico muro que separa a ricos y pobres en Lima. BBC Mundo.

Redclift, M. (2014). Development and the environmental crisis. Red or Green Alternatives. New York, USA: Routledge.

Sim, S. (2010). What the financial and environmental crisis is really telling us. Edinburgh, UK: Edinburgh University Press.

Vallaeys, F. (2014). La responsabilidad social universitaria: un nuevo modelo universitario contra la mercantilización. Revista lberoamericana de Educación Superior, 12(5), 105-117. Obtenido de http://www.sciencedirect.com/science/ journal/20072872/5/12

World Bank. (2007). Republic of Peru - Environmental Sustainability: A Key to Poverty Reduction in Peru. Washington, DC. Obtenido de https://openknowledge.worldbank.org/ handle/10986/7761 